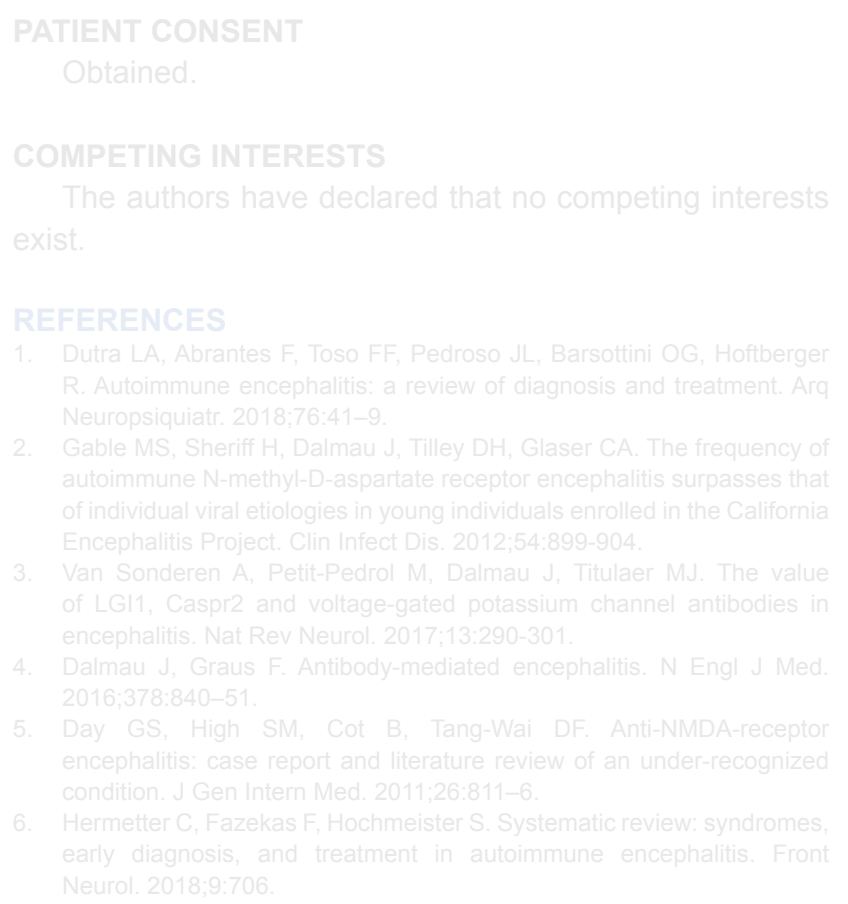

\title{
Intoxicação por Lítio após Cirurgia Bariátrica: Um Caso Clínico
}

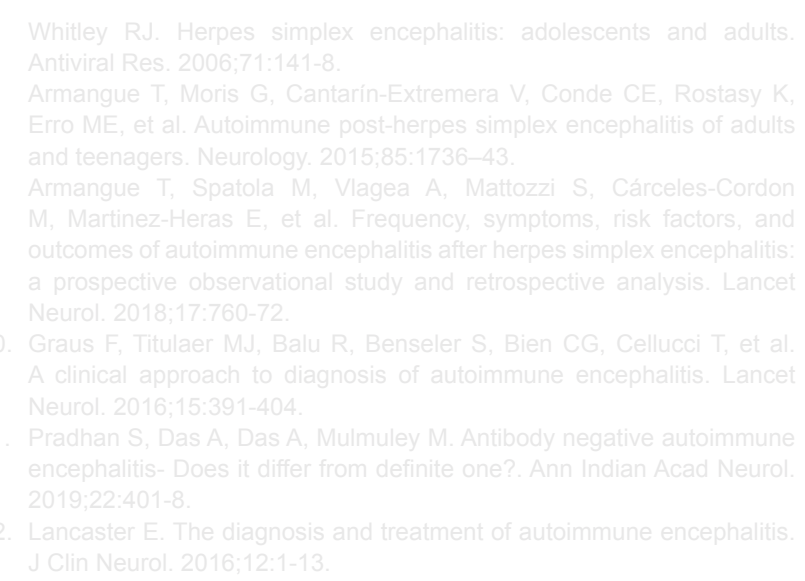

\section{Lithium Intoxication after Bariatric Surgery: A Case Report}

André Ribeirinho MARQUES ${ }^{1}$, António ALHO ${ }^{1}$, João Malhadas MARTINS ${ }^{1}$, Margarida BAIRRÃO1

Acta Med Port 2021 May;34(5):382-386 - https://doi.org/10.20344/amp.12868

\section{RESUMO}

A cirurgia bariátrica é uma opção terapêutica no tratamento da obesidade em doentes cuidadosamente selecionados, com perturbação psiquiátrica. Cerca de metade dos doentes referenciados para cirurgia bariátrica têm diagnosticada, pelo menos, uma perturbação mental, estando a maioria medicada com psicofármacos. Este procedimento pode alterar significativamente a biodisponibilidade dos fármacos e o lítio não é exceção. Contudo, apesar da absorção parecer diminuir na maioria dos fármacos, no caso do lítio existe um elevado risco de toxicidade. Neste artigo, descreve-se o caso de uma doente de 44 anos com um quadro de intoxicação por lítio após cirurgia bariátrica. Realizou-se uma revisão dos casos clínicos descritos na literatura de toxicidade ao lítio pós-cirurgia bariátrica e apresentam-se potenciais soluções clínicas preventivas. É essencial uma maior consciencialização das alterações na absorção dos psicofármacos pós-cirurgicos, particularmente no caso do lítio. Recomenda-se fortemente uma monitorização, de forma mais regular, pós-cirúrgica clínica e laboratorial dos níveis séricos de lítio.

Palavras-chave: Cirurgia Bariátrica; Lítio/efeitos adversos; Lítio/intoxicação

\section{ABSTRACT}

Bariatric surgery is a therapeutic option to treat obesity in (carefully selected) patients with psychiatric disorders. About half of the patients referred for bariatric surgery have a diagnosis of (at least one) mental disorder and most of them are treated with psychotropic drugs. This procedure may modify the bioavailability of drugs and lithium is no exception. However, although absorption seems to decrease in most drugs, in the case of lithium, there is a high risk of toxicity. In this article, we describe the case of a 44-year-old female patient with lithium intoxication after bariatric surgery. We conducted a review of the published clinical cases in the scientific literature about lithium toxicity after bariatric surgery, and we propose potential preventive clinical solutions. It is essential to increase awareness of changes to the absorption of psychotropic drugs in the post-surgery period, particularly in the case of lithium. Regular postoperative clinical and laboratory monitoring of lithium serum levels is strongly recommended.

Keywords: Bariatric Surgery; Lithium /adverse effects; Lithium/poisoning

1. Departamento de Psiquiatria e Saúde Mental. Hospital Distrital de Santarém. Santarém. Portugal.

$\triangle$ Autor correspondente: André Ribeirinho Marques. andreribeirinho@hotmail.com

Recebido: 24 de setembro de 2019 - Aceite: 13 de novembro de 2019 - First published: 16 de setembro de 2020- Online issue published: 03 de maio de 2021

Copyright $\odot$ Ordem dos Médicos 2021 


\section{INTRODUÇÃO}

A cirurgia de bypass gástrico tornou-se, nas últimas décadas, um tratamento cada vez mais frequente da obesidade. ${ }^{1} \mathrm{~A}$ obesidade é uma doença crónica com múltiplas consequências médicas, sendo que para alguns doentes, dieta e exercício físico, bem como a utilização de fármacos psicotrópicos não associados a aumento de peso, não são uma opção suficiente ou viável. ${ }^{2}$ São muitos os fatores que podem contribuir para a obesidade, sendo que no caso de pessoas com perturbação mental, o aumento de peso pode estar associado a alterações no estilo de vida (sedentarismo pela avolia, anedonia e isolamento social), alterações da dieta habitual (hiperfagia para pseudocompensação do humor, ou alterações da preferência alimentar), a efeitos adversos dos psicofármacos, entre outros. ${ }^{3} \mathrm{~A}$ cirurgia bariátrica apresenta-se, nestes doentes, como uma alternativa de tratamento da obesidade e consequentes comorbilidades médicas. ${ }^{2}$

A prevalência de pelo menos uma perturbação psiquiátrica em doentes referenciados para cirurgia bariátrica é significativa $(62,2 \%)$, sendo frequente a perturbação depressiva e/ou perturbação de ingestão compulsiva. $\mathrm{O}$ tratamento psicofarmacológico é comum, principalmente com antidepressivos. ${ }^{3}$ Dado que a cirurgia bariátrica provoca alterações anatómicas e fisiológicas que poderão ter impacto na absorção dos fármacos, é essencial para os clínicos co- nhecerem quais as consequências na farmacocinética dos mesmos após este procedimento cirúrgico. ${ }^{4}$

O lítio é um dos tratamentos de primeira linha na perturbação afetiva bipolar e com eficácia comprovada em outras doenças mentais. Porém, a sua janela terapêutica é estreita e a sua farmacocinética sofre modificações após a cirurgia bariátrica que implicam um acompanhamento multidisciplinar.

Neste caso clínico, descreve-se o caso de uma doente com perturbação afetiva bipolar, estabilizada há vários anos com lítio, que é admitida por um quadro clínico compatível com intoxicação grave ao lítio, um mês após bypass gástrico. Apresenta-se também uma revisão da escassa literatura existente sobre os mecanismos subjacentes, desafios e potenciais soluções.

\section{CASO CLÍNICO}

Mulher de 44 anos com antecedentes médicos de perturbação afetiva bipolar, diabetes mellitus tipo II, dislipidémia, hipertensão arterial e obesidade mórbida, tendo sido submetida um mês antes da observação em serviço de urgência (SU) a uma cirurgia bariátrica (técnica de bypass gástrico Roux-en-Y). Na observação em SU apresentava um quadro clínico com uma semana de evolução de agravamento progressivo, caracterizado por confusão mental,

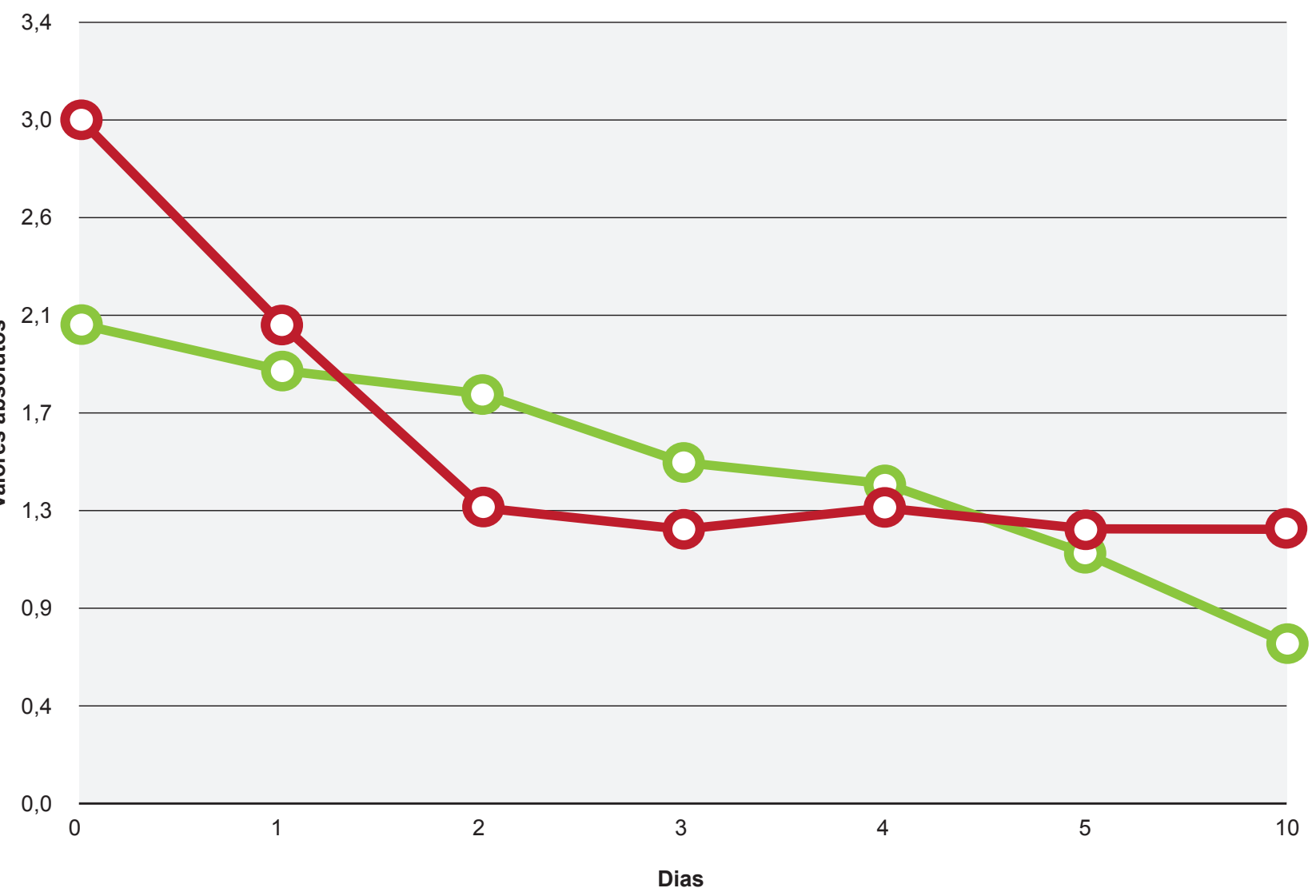

Figura 1 - Evolução dos valores de litémia e função renal, após suspensão de lítio e fluidoterapia 
fadiga persistente, fraqueza muscular marcada, tremores exuberantes dos membros superiores, dificuldades na coordenação motora, náuseas e diarreia. A medicação psicotrópica habitual diária, revista seis meses antes, seria quetiapina $300 \mathrm{mg}$, fluoxetina $20 \mathrm{mg}$, lorazepam $5 \mathrm{mg} \mathrm{e}$ carbonato de lítio 600 mg de libertação prolongada (litémia habitual $0,7 \mathrm{mmol} / \mathrm{L}$, terapêutica crónica há oito anos). No exame físico apresentava-se hemodinamicamente estável, apirética, salientando-se no exame neurológico sumário ataxia e hiperreflexia. A doente encontrava-se obnubilada, desorientada no tempo e espaço, com atenção captável mas facilmente dispersável e marcada perseveração ideativa, incapaz de colaborar na realização do restante exame mental. Analiticamente, salientava-se insuficiência renal aguda (creatinina $3,0 \mathrm{mg} / \mathrm{dL}$, ureia $130 \mathrm{mg} / \mathrm{dL}$ ) e hiponatrémia (128 mEq/L).

Após doseamento de litémia, que se apresentava em níveis de 2,1 $\mathrm{mmol} / \mathrm{L}$ (toxicidade $>1,5 \mathrm{mmol} / \mathrm{L}$ ), assumiu-se quadro de intoxicação por lítio e foi internada no serviço de Medicina Interna. Optou-se por abordagem conservadora inicial (suspensão de lítio e hidratação por fluidoterapia), que se manteve pela rápida e progressiva melhoria clínica e analítica, em cinco dias, como verificado na Fig. 1.

Por manutenção de níveis de creatinina no limite superior normal (valores de referência: 0,6 a $1,1 \mathrm{mg} / \mathrm{dL}$ ) e dificuldade na depuração total de lítio, optou-se pela não reintrodução do fármaco, com manutenção de humor eutímico e sem oscilações da energia vital.

\section{DISCUSSÃO}

A cirurgia bariátrica provoca alterações anatómicas e fisiológicas que poderão ter impacto na absorção oral dos fármacos. Os procedimentos que provoquem mal-absorção (bypass e derivação biliopancreática) têm maior impacto na farmacocinética dos fármacos, destacando-se como alterações farmacocinéticas características a redução da acidez gástrica, a redução do volume e motilidade do estômago, esvaziamento gástrico e trânsito intestinal acelerados. ${ }^{4}$

Um estudo in vitro, mostrou que a dissolução de fármacos estaria reduzida em 10 de 22 psicofármacos testados, porém no lítio, esta taxa estaria aumentada em mais de $200 \%$. 5

O lítio é rápida e totalmente absorvido no estômago e intestino proximal. É hidrofílico e não se liga a proteínas, não sofre metabolização, sendo excretado pelo rim praticamente na totalidade. Níveis séricos entre os 0,6 e 1,0 $\mathrm{mmol} / \mathrm{L}$ são considerados terapêuticos na pperturbação afetiva bipolar (doses mais altas, entre 1,0 e 1,2 mmol/L, estão indicadas para tratamento e prevenção da mania aguda), sendo considerados tóxicos quando acima de 1,5 $\mathrm{mmol} / \mathrm{L}$ (toxicidade ligeira: 1,5 - 2,5 mmol/L; toxicidade moderada: 2,5 - 3,5 mmol/L; toxicidade grave: > 3,5 mmol/L). ${ }^{7,8}$ Os sintomas de toxicidade associada ao lítio são descritos na Tabela $1 .^{7}$

Dentro dos fatores de risco principais para toxicidade temos: interações medicamentosas (anti-inflamatórios não esteroides, diuréticos e IECA/ARA), idade avançada, doença crónica e dose total diária de lítio. ${ }^{9}$

A intoxicação por lítio, nestes casos, é essencialmente provocada pelas reduções de volume de água e massa corporal: a redução de aporte líquido provoca desidratação e, consequente lesão renal aguda, diminuindo a depuração de lítio; no caso particular do carbonato de lítio, o menos solúvel de todos os sais de lítio, tais alterações levam à sua acumulação e absorção prolongada; verifica-se também que, no geral, doentes obesos apresentam aumento da capacidade de depuração de lítio. ${ }^{10-15}$

Neste caso clínico, optou-se por uma abordagem

Tabela 1 - Manifestações clínicas da intoxicação por lítio ${ }^{6}$

Neuropsiquiátricas

Tremor, apatia, sonolência, agitação, disartria, ataxia, nistagmo, movimentos coreiformes, hiperreflexia, mioclonias, confusão mental, convulsões, coma.

Cardíacas

Alterações do ECG do tipo 'wandering atrial pacemaker', bradicardia sinusal, supra- e infradesnivelamento do segmento ST, inversão da onda T, QT longo.

Gastrointestinais Náuseas, vómitos, dor abdominal, distensão abdominal e diarreia.

Tabela 2 - Recomendações para hemodiálise na intoxicação a lítio8

Indicações

Hemodiálise é recomendada:

- Se insuficiência renal e litémia $>4,0 \mathrm{mmol} / \mathrm{L}$;

- Se diminuição do nível de consciência, convulsão, ou arritmia grave (independentemente dos níveis séricos de lítio).

Hemodiálise é sugerida:

- Se litémia > 5,0 mmol/L;

- Se síndrome confusional;

- Se tempo estimado para alcançar litémia $<1,0 \mathrm{mmol} / \mathrm{L}$, em condições ótimas, for $>36$ horas.

Interrupção

- Quando litémia < 1,0 mmol/L ou evidência de melhoria clínica;

- Após um mínimo de 6 horas de hemodiálise, na ausência de resultado de doseamento sérico.

Realizar doseamentos seriados durante 12 horas, após interrupção, para determinar continuidade de tratamento. 
Tabela 3 - Recomendações para monitorização dos níveis de lítio ${ }^{15}$

\begin{tabular}{ll}
\hline Fase peri-operatória & Recomendações \\
\hline $\begin{array}{l}\text { Pré-operatória } \\
\text { (durante período de dieta líquida) }\end{array}$ & Doseamento semanal \\
Pós-operatório ( 0 - 6 semanas) & Doseamento semanal \\
Pós-operatório (> 6 semanas) & Doseamento quinzenal até aos 6 meses \\
& Dosemento mensal após 6 meses \\
& Doseamento na regularidade habitual após um ano \\
Se os níveis séricos aumentarem mais de $25 \%$ ou se aproximarem de 1,2 mmol/L, considerar reduzir a dose. \\
$\begin{array}{l}\text { Se sinais de toxicidade, rever dose. } \\
\text { Avaliar estado mental periodicamente, recorrendo a escalas formais se possível. } \\
\text { Incentivar reforço hídrico oral }(2,5-3 \text { L/dia) na fase pré-operatória. }\end{array}$ \\
\hline
\end{tabular}

conservadora, ainda que a hemodiálise fosse uma abordagem possível e frequentemente necessária. As recomendações para hemodiálise são apresentadas na Tabela $2 .{ }^{8}$

Ainda que a toxicidade seja a preocupação mais importante nestes doentes, níveis subterapêuticos podem também ter consequências significativas em doentes com perturbações do humor graves. Dada a imprevisibilidade da absorção e outras alterações farmacocinéticas, os níveis subterapêuticos são também teoricamente possíveis, uma vez que a absorção do lítio se encontra condicionada pela redução de intestino funcional. ${ }^{15}$

O caso clínico apresentado alerta-nos para as particularidades de uma população especial de doentes. Ainda são raros os casos descritos na literatura científica internacional de intoxicações iatrogénicas a psicofármacos após cirurgia bariátrica. Uma proporção significativa de doentes que são submetidos a este tipo de intervenção sofrem de doença psiquiátrica, podendo a etiologia da obesidade (inevitavelmente multifatorial) ser consequência da doença e/ou dos efeitos iatrogénicos do tratamento. Assim sendo, e perante algum grau de desconhecimento e imprevisibilidade ainda existente relativamente ao grau de absorção dos psicofármacos, é recomendável um acompanhamento regular e uma boa articulação entre as equipas de cirurgia bariátrica e psiquiatria na gestão destes casos.

Não existem, até à data, guidelines baseadas em evidência científica publicadas relativamente à abordagem de doentes sob lítio, sujeitos a cirurgia bariátrica. Porém, baseado nos dados da literatura e na opinião de peritos, algumas recomendações podem ser feitas, designadamente, uma monitorização pós-cirúrgica clínica e laboratorial dos níveis de lítio sérico mais regular, como se pode verificar na Tabela $3 .{ }^{15}$ Mais investigação sobre as alterações farmacocinéticas após este tipo de intervenções, nomeadamente a longo prazo, são necessárias para um acompanhamento clínico sustentado em maior evidência.

\section{PROTEÇÃO DE PESSOAS E ANIMAIS}

Os autores declaram que os procedimentos seguidos estavam de acordo com os regulamentos estabelecidos pelos responsáveis da Comissão de Investigação Clínica e Ética e de acordo com a Declaração de Helsínquia da Associação Médica Mundial publicada em 2013.

\section{CONFIDENCIALIDADE DOS DADOS}

Os autores declaram ter seguido os protocolos do seu centro de trabalho acerca da publicação de dados.

\section{CONSENTIMENTO DO DOENTE}

Obtido.

\section{CONFLITOS DE INTERESSE}

Os autores declaram não ter conflitos de interesses relacionados com o presente trabalho.

\section{FONTES DE FINANCIAMENTO}

Este trabalho não recebeu qualquer tipo de suporte financeiro de nenhuma entidade no domínio público ou privado.

lithium après chirurgie bariatrique. Rev Med Liege. 2018;73:82-7

7. NICE. Bipolar disorder: assessment and management. Clinical Guideline no. 185. London: NICE; 2014 [consultado 2019 nov 6]. Disponível em: http://www.nice.org.uk/

8. Decker BS, Goldfarb DS, Dargan PI, Friesen M, Gosselin S, Hoffman $\mathrm{RS}$, et al. Extracorporeal treatment for lithium poisoning: systematic review and recommendations from the EXTRIP workgroup. Clin J Am Soc Nephrol. 2015;10: 875-87.

9. Heath LJ, Billups SJ, Gaughan KM, Gardner KN, Dugan D, Botts SR. Risk factors for utilization of acute care services for lithium toxicity. Psychiatric Services. 2018;69:671-6.

10. Reiss RA, Haas CE, Karki SD, Gumbiner B, Welle SL, Carson SW. Lithium pharmacokinetics in the obese. Clin Pharmacol Ther. 1994; 55:392-8.

11. Tripp AC. Lithium toxicity after Roux en $Y$ gastric bypass surgery. J Clin 
Psychopharmacol. 2011;31:261-2.

12. Musfeldt D, Levinson A, Nykiel J, Carino G. Lithium toxicity after Roux en $Y$ bariatric surgery. BMJ Case Rep. 2016;2016.

13. Walsh K, Volling J. Lithium toxicity following Roux en $Y$ gastric bypass. Bariatr Surg Pract Patient Care. 2014;9:77-80.

14. Alam A, Raouf S, Recio FO. Lithium toxicity following vertical sleeve gastrectomy: a case report. Clin Psychopharmacol Neurosci. 2016;14:318-20.

15. Bingham KS, Thoma J, Hawa R, Sockaling S. Perioperative lithium use in bariatric surgery: a case series and literature review. Psychosomatics. 2016; 57:638-44 